\title{
Global SUSY Fits with the MasterCode Framework COLLABORATION
}

\section{Samuel ROGERSON*Imperial College London}

E-mail: samr@cern.ch

We present the latest results of the MasterCode collaboration on global SUSY fits. Currently available experimental data are used to determine the preferred SUSY and Higgs boson mass scales. The data comprise a combination of high-energy SUSY searches, low-energy precision measurements and astrophysical data. We include all relevant LHC searches for SUSY, electroweak precision observables such as the $\mathrm{W}$ boson mass and the anomalous magnetic moment of the muon, B physics observables such as $\operatorname{BR}(b \rightarrow s \gamma)$, as well as the cold dark matter density in the Universe. The preferred masses for SUSY particles as well as for the MSSM Higgs bosons are derived in the context of four GUT-based realizations of the MSSM. We find a preference for relatively light SUSY masses, which the direct searches at the LHC shift to slightly higher mass scales. The preferred mass values can directly be compared to the reach of the LHC and future $e^{+} e^{-}$colliders as well as to current and future direct detection searches for dark matter.

XXIst International Europhysics Conference on High Energy Physics

21-27 July 2011

Grenoble, Rhônes Alpes France

\footnotetext{
* Speaker.
} 


\section{Introduction}

We extend our previous[1] frequentist analyses of the CMSSM, NUHM1, VCMSSM and mSUGRA parameter spaces taking into account all the public results of searches for supersymmetry using data from the 2010 LHC run and the Xenon100 direct search for dark matter scattering. The LHC data set includes ATLAS and CMS searches for jets $+H_{T}$ events (with or without leptons) and for the heavier MSSM Higgs bosons, and the upper limit on $\operatorname{BR}\left(B_{s} \rightarrow \mu^{+} \mu^{-}\right)$including data from $\mathrm{LHCb}$ as well as CDF and DØ.

\section{Experimental Results}

\subsection{ATLAS and CMS}

We incorporate the CMS $E_{T}$ [2] and the ATLAS $0 \ell$ and $1 \ell[3]$ results, each using $\sim 35 \mathrm{pb}^{-1}$ of data. The limits obtained in both searches are very close to the median expected limit, corresponding to a difference between the numbers of events observed and expected from background that is negligible compared to the $\sigma_{\text {eff }}$ for the number of background events. We therefore approximate the impact of these searches outside their nominal 95\% CL contour by assuming that the number of effective $\sigma$ is simply proportional to the number of signal events expected at any given supersymmetric point, which we assume to be $\sim M^{-4}$ and we then calculate the corresponding $\chi^{2}$ penalty as

$$
\chi_{p}^{2}=\chi_{95 \%}^{2}\left(\frac{M_{p}}{M_{95 \%}}\right)^{4}
$$

For each point in parameter space, we take the contribution arising from the search with the maximum expected exclusion.

\subsection{Heavy Higgs}

The CMS Collaboration has provided model-independent limits on the H/A production cross section times $\tau^{+} \tau^{-}$branching ratio $(\sigma \times B R)$ at the $68 \%, 95 \%$ and $99.7 \% C L_{s}$ as functions of $M_{A}$ [4], corresponding to a one-dimensional $\chi^{2}$ contribution of $1,3.84$, and 9 , respectively. For each fixed value of $M_{A}$, we assume that the $\chi^{2}$ penalty for other values of $\sigma \times B R$ may be approximated by the functional form $\Delta \chi 2 \propto(\sigma \times B R)^{p\left(M_{A}\right)}$, normalized to unity on the $68 \%$ CL line and fitting the power $p\left(M_{A}\right)$ independently for each value of $M_{A}$ (typical values are $\sim 1.3$ ). Assuming a scaling law of $(\sigma \times B R) \propto \tan ^{2} \beta$, we then apply a $\chi^{2}$ penalty calculated as

$$
\chi^{2} \sim\left(\frac{\tan ^{2} \beta}{\tan ^{2} \beta_{95 \%}}\right)^{p\left(M_{A}\right)}
$$

\section{$2.3 \mathrm{BR}\left(B_{s} \rightarrow \mu^{+} \mu^{-}\right)$}

The paper by $\mathrm{LHCb}[5]$ provides $95 \%$ and $90 \%$ upper limits on $\operatorname{BR}\left(B_{s} \rightarrow \mu^{+} \mu^{-}\right)$of 56 and $43 \times 10^{-9}$, to be compared with the Standard Model prediction of $(3.2 \pm 0.2) \times 10^{-9}$. A combination of these with the results provided by CDF [6] and DØ [7] is achieved by first performing approximate studies, based on the signal and background expectations in each experiment, and comparing with the observed pattern of events, generating toy experiments that reproduce their 
quoted 90\% CL upper limits. The toy LHCb experiment was constructed using the information shown in Table 3 of [5]. The toy CDF experiment was based on the information given in Table II of [6], combined with the invariant mass resolution, normalization factors and averaged Neural Network efficiencies quoted in the text. Finally, the toy DØ experiment was based on Fig. 4 of [7], together with the invariant mass resolution and normalization factor quoted in the text. The results of the three experiments were combined using the $C L_{s}$ method. Our global fit uses the full likelihood function calculated using the above experimental information to beyond the 99\% CL.

\subsection{Xenon100 search for dark matter scattering}

Finally, we implement the constraint imposed by the direct upper limit on dark matter scattering given by the Xenon 100 experiment [8]. This takes the form of a 95\% CL upper limit on the spin-independent cross section as a function of $m_{\tilde{\chi}_{1}^{0}}$. The Xenon100 Collaboration report the observation of 3 events where $1.8 \pm 0.6$ events were expected. Using Poisson statistics with a nonnegligible background, we have constructed a model for the Xenon100 contribution to the global $\chi^{2}$ likelihood function as a function of the number of events using the $C L_{s}$ method. This turns out to be quite similar to a Gaussian function with mean 1.2 and standard deviation 3.2 events. Our model for the Xenon100 likelihood function yields a 90\% CL upper limit of 6.1 events so, for any given value of $m_{\tilde{\chi}_{1}^{0}}$, we assume that the $90 \%$ CL upper limit on $\sigma_{p}^{\text {SI }}$ quoted in [8] corresponds to 6.1 events, and use simple scaling to estimate the event numbers corresponding to other values of $\sigma_{p}^{\text {SI }}$. We then use the Gaussian model for the Xenon $100 \chi^{2}$ function to estimate the contribution of this experiment to the global likelihood function for other $\sigma_{p}^{\text {SI }}$ values. We note that, because of the insignificant 'excess' of 1.2 events in the Xenon100 data, there is a contribution $\Delta \chi^{2} \sim 0.3$ to the global likelihood function at small values of $\sigma_{p}^{\mathrm{SI}}$

We take account of the uncertainty in the calculation of $\sigma_{p}^{\text {SI }}$ induced principally by the experimental uncertainty in the $\pi$-nucleon $\sigma$ term, $\Sigma_{\pi N}$. Estimates of $\Sigma_{\pi N}$ up to a value as large as $64 \pm 8 \mathrm{MeV}$ have been given in the literature [9]. Here we span the plausible range by using as our default $\Sigma_{\pi N}=50 \pm 14 \mathrm{MeV}$, while also showing some results for $\Sigma_{\pi N}=64 \pm 8 \mathrm{MeV}$

\section{Results}

We see that for the constrained models of supersymmetry considered, the direct searches from CMS/ATLAS push the best fit points, Table 1 , in $m_{1 / 2}$ to $\sim 500 \mathrm{GeV}$ corresponding to $m_{\tilde{g}} \gtrsim 1 \mathrm{TeV}$. The CMS limits on heavy Higgs production and our compilation of LHCb, CDF and DØ constraints on $\mathrm{BR}\left(B_{s} \rightarrow \mu^{+} \mu^{-}\right)$have impacts on the parameter spaces of the NUHM1, but do not affect significantly the favoured regions of the CMSSM, VCMSSM and mSUGRA. The Xenon100 results have an impact on the model parameter spaces that would be significant if $\Sigma_{\pi n}$ were large, $\sim 60 \mathrm{MeV}$. However, the current uncertainty in $\Sigma_{\pi n}$ does not permit a strong conclusion to be drawn.

Overall, we calculate probabilities in the constrained models after the search exclusions of $<20 \%$, Table 1 and Figure 1. With the advent of direct searches using $1 \mathrm{fb}^{-1}$ of data one might expect to see $p<5 \%$ if no signal is seen. This not only applies to direct searches using $\mathbb{E}_{T}$ with (or without) leptons, but also the indirect searches through processes like $\operatorname{BR}\left(B_{S} \rightarrow \mu^{+} \mu^{-}\right)$where these models have preferred values $\sim \mathrm{SM}$. 
This indicates a slight tension in these models between the preference for rather light colourneutral states arising in particular from $(g-2)_{\mu}$ and the search limits from the direct searches for coloured SUSY particles at the LHC. The mSUGRA scenario yields a significantly worse description of the data than the other considered models already for the pre-LHC data set, and inclusion of the 2010 LHC and Xenon100 constraints has only a small impact on the preferred fit values and the fit probabilities. If the upcoming LHC results lead to a further increase of the excluded mass regions for coloured superpartners, the CMSSM, NUHM1 and VCMSSM scenarios could eventually get under pressure. Such a tension could be avoided in realisations of SUSY with a larger splitting between the coloured and the colour-neutral part of the spectrum (for instance in GMSB-type scenarios), such that the masses of squarks and gluinos are in the $\mathrm{TeV}$ range, while sleptons, neutralinos and charginos can still be light.

\begin{tabular}{|r||c|c|c|c|c|c||c|}
\hline Model & Min $\chi^{2}$ & Prob & $m_{1 / 2}$ & $m_{0}$ & $A_{0}$ & $\tan (\beta)$ & $M_{h}^{\text {no LEP }}$ \\
\hline \hline CMSSM & $22.5 / 19$ & $26 \%$ & 310 & 60 & -60 & 10 & 109 \\
post-LHC/Xenon & $26.2 / 20$ & $16 \%$ & 470 & 170 & -780 & 22 & 116 \\
\hline NUHM1 & $20.5 / 17$ & $25 \%$ & 240 & 100 & 920 & 7 & 119 \\
post-LHC/Xenon & $24.2 / 19$ & $19 \%$ & 530 & 110 & -370 & 27 & 118 \\
\hline
\end{tabular}

Table 1: The best fit values of the four models considered
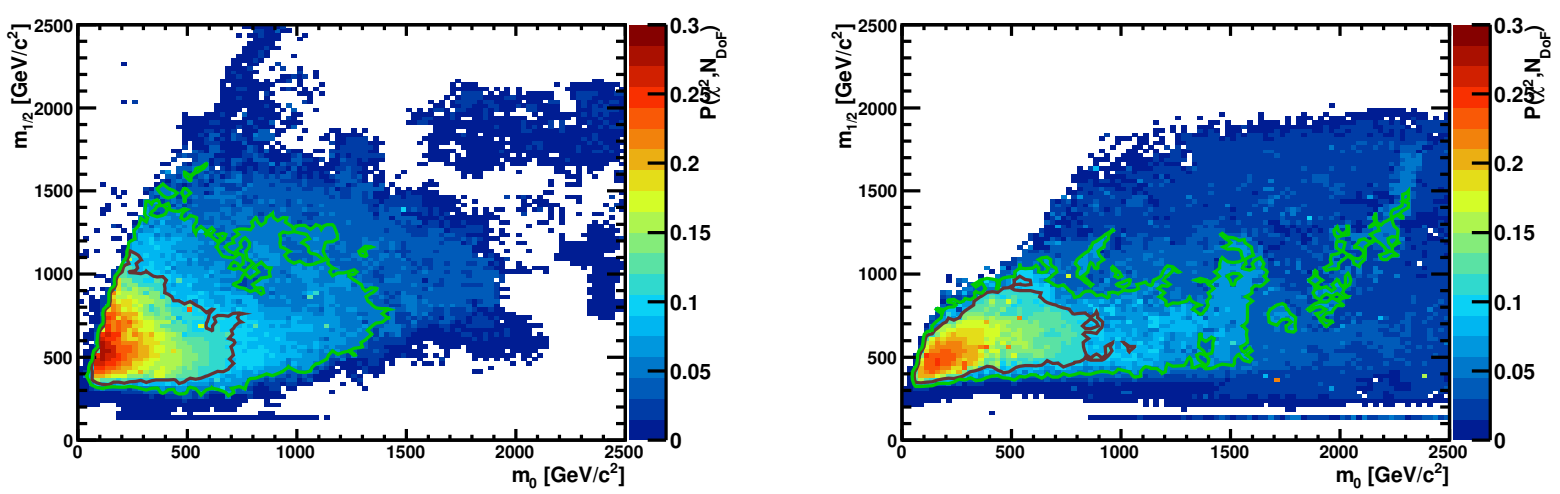

Figure 1: The $\left(m_{0}, m_{1 / 2}\right)$ planes in the NUHM1 (left) and the CMSSM (right), for the after applying the various experimental constraints. In each plane, different regions are colour-coded according to the p-values found in our global fits. We note that in the $\mathrm{LHC}_{2010}$ analysis the regions with $p>0.05$ extend up to $m_{1 / 2} \sim 1500 \mathrm{GeV}$ and $2000 \mathrm{GeV}$ respectively 


\section{References}

[1] O. Buchmueller et al., Eur. Phys. J. C 71 (2011) 1634 [arXiv:1102.4585 [hep-ph]].

[2] V. Khachatryan et al. [CMS Collaboration], http://cdsweb.cern.ch/record/1343076/ files/SUS-10-005-pas.pdf; see also https://twiki.cern.ch/twiki/bin/view/ CMSPublic/PhysicsResultsSUS.

[3] G. Aad et al. [ATLAS Collaboration], http: / / cdsweb. cern. ch/record/1345745/ files/ATLAS-CONF-2011-064.pdf, https://atlas.web.cern.ch/Atlas/GROUPS / PHYSICS/CONFNOTES/ATLAS-CONF-2011-064/.

[4] V. Khachatryan et al. [CMS Collaboration], https://twiki.cern.ch/twiki/bin/view/ CMSPublic/PhysicsResultsHIG10002/

[5] R. Aaij et al. [LHCb Collaboration], arXiv:1103.2465 [hep-ex].

[6] T. Aaltonen et al. [CDF Collaboration], Phys. Rev. Lett. 100, 101802 (2008) [arXiv:0712.1708 [hep-ex]]; see also http://www-cdf.fnal.gov/physics/new/ bottom/090813.blessed-Bsd2mumu// bsmumupub3.7fb_v01.pdf.

[7] V. M. Abazov et al. [D0 Collaboration], Phys. Lett. B 693 (2010) 539 [arXiv:1006.3469 [hep-ex]].

[8] E. Aprile et al. [XENON100 Collaboration], arXiv:1104.2549 [astro-ph.CO].

[9] M. M. Pavan, I. I. Strakovsky, R. L. Workman and R. A. Arndt, PiN Newslett. 16 (2002) 110 [arXiv:hep-ph/0111066]. 